Inner products of resonance solutions in 1D quantum barriers

This article has been downloaded from IOPscience. Please scroll down to see the full text article.

2010 J. Phys. A: Math. Theor. 43175301

(http://iopscience.iop.org/1751-8121/43/17/175301)

View the table of contents for this issue, or go to the journal homepage for more

Download details:

IP Address: 161.111.23.135

The article was downloaded on $31 / 05 / 2010$ at 10:58

Please note that terms and conditions apply. 


\title{
Inner products of resonance solutions in 1D quantum barriers
}

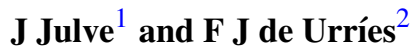 \\ ${ }^{1}$ IFF, Consejo Superior de Investigaciones Científicas, Serrano 113 bis, Madrid 28006, Spain \\ ${ }^{2}$ Departamento de Física, Universidad de Alcalá de Henares, Alcalá de Henares, Madrid, Spain \\ E-mail: julve@imaff.cfmac.csic.es and fernando.urries@uah.es
}

Received 17 November 2009, in final form 12 February 2010

Published 13 April 2010

Online at stacks.iop.org/JPhysA/43/175301

\begin{abstract}
The properties of a prescription for the inner products of resonance (Gamow states), scattering (Dirac kets) and bound states for one-dimensional quantum barriers are worked out. The divergent asypmtotic behaviour of the Gamow states is regularized using a Gaussian convergence factor first introduced by Zel'dovich. With this prescription, most of these states (with discrete complex energies) are found to be orthogonal to each other and to the Dirac kets, except when they are neighbours, in which case the inner product is divergent. Therefore, as it happens for the continuum scattering states, the norm of the resonant ones remains non-calculable. Thus, they exhibit properties halfway between the (continuum real) Dirac- $\delta$ orthogonality and the (discrete real) Kronecker- $\delta$ orthogonality of the bound states.
\end{abstract}

PACS number: 03.65.Nk

\section{Introduction}

Resonances in quantum mechanics describe states evolving non-unitarily [1], both decaying and building ones, and have found applications in the study of nuclear reactions. As such, they have been more often studied in the case of spherical nuclear potentials, mainly of the 'shell-model' type and restricting the analysis to the radial $s$-wave equation. Albeit for obvious unessential differences in the boundary conditions, the physical picture is similar to the case of the one-dimensional barriers we are considering, more akin to condensed matter systems or ion-trapping devices. There is ongoing interest and work still in progress on the physical interpretation of resonances [2].

Resonant solutions to the Schrödinger equation occur in any simple potential barrier with a compact support, the plain square barrier being the most simple, tractable and fully representative example, and they are more often found in alternative specialized expansions of the Green's function and other physically relevant objects. They correspond to complex 
energies and momenta, which place them out of the familiar realm of Hermitian operators in Hilbert spaces, where a wealth of well-known mathematical properties is available. Though having been first studied in early times [3, 4], the non-trivial mathematical properties of resonances have spurred a long lasting investigation effort. This paper focuses on some still debatable issues regarding the norm, inner products and completeness properties of resonant states.

The issue of the completeness arises in its simplest form as soon as one attempts, for instance, to expand the identity in terms of projectors on the bound (if any) $\left|\phi_{i}\right\rangle$, resonance $\left|z_{n}\right\rangle$ and background ('scattering' complex energy) $|z\rangle$ states, namely

$$
I=\sum_{i}\left|\phi_{i}\right\rangle\left\langle\phi_{i}\left|+\sum_{n}\right| z_{n}\right\rangle\left\langle z_{n}|+\oint \mathrm{d} z| z\right\rangle\langle z| .
$$

where the second sum may involve a variable number and type of resonances. Such an expansion is, in principle, attainable by deforming, in the complex (two-sheet) plane [5], the continuum real energy integration occurring in the traditional expansion in terms of bound and scattering states:

$$
I=\sum_{i}\left|\phi_{i}\right\rangle\left\langle\phi_{i}\left|+\int \mathrm{d} E\right| E\right\rangle\langle E|
$$

following the lines of the proof of (2), as given for instance by [6]. In particular cases, a complete expansion in terms of resonances (plus bound states) can be found, as for example for continuum wavefunctions (or the scattering solutions) within a finite region including the support of the barrier [7-9]. This has direct application for instance in time-dependent problems, as in the time evolution of quantum decay [10]. In the general case, covering the whole space, directly testing any resonance expansion like (1) against the idempotence requirement $I^{2}=I$, needs the computation of the inner products of all these families of states with each other. We aim to perform some steps in this direction.

The difficulty stems from the divergent asymptotic behaviour of the resonance solutions, which leads to infinite norms and seemingly divergent and hard to calculate inner products, and has given rise to a variety of proposals to circumvent it. These proposals adopt different prescriptions to render finite the space integrals involved in these products and in general matrix elements. We may quote analytical continuations of the resonant solutions in the complex momentum plane [11], the 'external complex scaling' [12] of the space-coordinate integration variable, and the introduction of convergence factors in the integrals.

In this paper we shall adopt a Gaussian convergence factor, first introduced by Zel'dovich [13] and used by others [5], with which a limit can be worked out yielding novel non-trivial results; in fact, we recover the result of the integrals that were already well defined, and extend the finite result to a newer region of the complex momentum plane. The procedure, which avoids relying on analytical continuation arguments, leads to a prescription for the definition of the inner products, yielding a specific set of orthogonality relations. Our results are derived for a general potential with finite support of which the bound and resonant poles of the $S$-matrix are known.

The usual inner product in the Hilbert space, involving the complex conjugate of one of the wavefunctions, is defined so as to have real probability densities and norms for the general wavefunctions. However when dealing with resonant solutions, a symmetrical inner product is involved in the convolution of the Green's function with initial states (superpositions of scattering states), and is associated with a complex 'norm' [14], so we explore the properties of both alternative definitions.

The plan of the paper is the following: In section 2, we briefly review the relevant features of the resonances while fixing some notation and stressing the relationships between proper, 
anti-, outgoing and incoming resonant solutions. In section 3, we calculate the standard inner product of resonant states between themselves and with the bound and the scattering states. In section 4, we introduce the symmetrical inner product, and give the result for the resonant-resonant and resonant-scattering product. Then in section 5, we outline in some detail the regularization prescription adopted, based on a Gaussian factor, and show how the (either finite or divergent) results are recovered in the non-regularized limit. For comparison, we comment here on some other prescriptions found in the literature. Finally, the conclusions are drawn in section 6 . The crucial integral formulae are derived in the appendix.

\section{The resonant solutions}

We consider the 1D time-independent Schrödinger equation

$$
\left[\frac{\partial^{2}}{\partial x^{2}}+p^{2}-2 m V(x)\right] \psi(x, p)=0
$$

where we use units such that $\hbar=1$.

For a cut-off potential $V(x)$ describing a general barrier with support in the compact interval $[0, L]$, besides the usual scattering in and out solutions for continuous real energy $E=p^{2} / 2 m>0$ and possible bound states (discrete real $E_{i}<0$ ), one has resonant solutions (Gamow states) satisfying the homogeneous outgoing resonant boundary conditions (ORBCs),

$$
\left.\partial_{x} \psi\right|_{x=0}=-\mathrm{i} p \psi(0),\left.\quad \partial_{x} \psi\right|_{x=L}=\mathrm{i} p \psi(L)
$$

The solutions $u_{n}(x)$, called proper (outgoing) resonances, exist for a denumerable set of isolated values $p_{n}$ of $p$ (with corresponding energies $z_{n}=p_{n}^{2} / 2 m$ ) lying inside the octants close to the real axis (i.e. $\left|\operatorname{Re} p_{n}\right|>\left|\operatorname{Im} p_{n}\right|$ ) in the lower half complex plane (i.e. $\operatorname{Im} p_{n}<0$ ), and occupy symmetrical positions with respect to the imaginary axis (see figure 1). It is customary to label them as $p_{n}(n=1,2, \ldots)$ when $\operatorname{Re} p_{n}>0$ and as $p_{-n} \equiv-p_{n}^{*}$ their symmetric ones, sometimes called anti-resonances. In the case of a simple square barrier, the real parts $\operatorname{Re} p_{n}$ tend to be spaced regularly for increasing $|n|$, while $\left|\operatorname{Im} p_{n}\right|$ grows slowly [1].

RBCs with reversed sign of $p$ in (4) correspond to incoming solutions $\tilde{u}_{n}(x)$, and the momenta $\tilde{p}_{n} \equiv-p_{n}=p_{-n}^{*}$ lie in the upper half complex plane. We denote with $\tilde{u}_{n}(x)=u_{n}\left(\tilde{p}_{n} ; x\right)$ these solutions and with $\left|\tilde{z}_{n}\right\rangle$ the corresponding states. Note that the corresponding energies $\tilde{z}_{n}$ lie in the first Riemann sheet. For real potentials $V(x)$, the complex conjugates $u_{n}^{*}(x)$ of the outgoing resonances correspond instead to yet outgoing solutions with mirror momenta $-p_{n}^{*}$, and energies $z_{n}^{*}$ in the second Riemann sheet, so that $u_{n}^{*}(x)=u_{-n}(x)$ and $z_{n}^{*}=z_{-n}$. For the same reason, the complex conjugates of the incoming resonances are again incoming solutions. In the literature, $u_{n}^{*}(x)$ have been easily mistaken for an incoming solution.

The ORBCs are equivalent to imposing the asymptotic form

$$
u_{n}(x)= \begin{cases}R_{n} \mathrm{e}^{-\mathrm{i} p_{n} x}, & x \leqslant 0 \\ T_{n} \mathrm{e}^{\mathrm{i} p_{n} x}, & x \geqslant L\end{cases}
$$

where the amplitudes $R_{n}$ and $T_{n}$ differ by a phase and are defined up to a global arbitrary factor. An immediate consequence is that the norm

$$
\left\|u_{n}\right\| \equiv\left\langle z_{n} \mid z_{n}\right\rangle=\int_{-\infty}^{+\infty} \mathrm{d} x u_{n}^{*}(x) u_{n}(x) \approx \int_{-\infty}^{+\infty} \mathrm{d} x \exp 2|x|\left|\operatorname{Im} p_{n}\right|=\infty
$$

is even more divergent than for the Dirac states $\|\psi\| \equiv\langle E \mid E\rangle=\delta(0) \approx \int_{-\infty}^{+\infty} \mathrm{d} x=\infty$, causing both kinds of states not to belong to $\mathcal{L}^{2}$.

This fact, as opposite to the finite norm of the bound states, has been addressed in the framework of rigged Hilbert spaces (RHS) [15, 16] along the same lines adopted for the Dirac 
kets. In RHS, these states are interpreted just as linear functionals on more restricted spaces (Hilbert or Schwartz) in which an inner product is properly defined, but nevertheless it is customary to consider, in a somewhat relaxed sense, inner products of the Dirac kets which have a meaning only as distributions, namely $\left\langle E \mid E^{\prime}\right\rangle=\delta\left(E-E^{\prime}\right)$. However, when one tries to give an answer of this type for the resonance states, the inner products

$$
\left\langle z_{n} \mid z_{m}\right\rangle=\int_{-\infty}^{+\infty} \mathrm{d} x u_{n}^{*}(x) u_{m}(x)
$$

may be expected to be generally divergent as well, because of the exponential growth for large $x$. For this reason, their actual calculation has spurred the adoption of a number of elaborated strategies.

The same difficulties affect the inner products $\left\langle z_{n} \mid E\right\rangle$ between resonant and scattering states and, more generally, between resonant states and wave packets. This is an important issue as long as the analytical calculation of, for instance, the time evolution of an initial state impinging on a barrier [9], or of the shutter problem [17], contains sums of terms with poles in the resonant momenta. For the (either Laplace or Fourier) transform of the time-dependent Green's function, one has [14]

$$
G\left(x, x^{\prime} ; p\right)=\sum_{n} \frac{1}{N_{n}} \frac{1}{2 p_{n}} \frac{u_{n}(x) u_{n}\left(x^{\prime}\right)}{p-p_{n}}+\text { regular terms. }
$$

The interpretation of the pole terms as (transient) excitations of the resonant eigenmodes of the system requires the knowledge of the projection of the initial state on the resonant ones. The dimensionless normalization factor in $(8)$ is $[14,17]$

$$
N_{n}=\mathrm{i} \frac{u_{n}^{2}(a)+u_{n}^{2}(b)}{2 p_{n}}+\int_{a}^{b} \mathrm{~d} x u_{n}^{2}(x),
$$

where $a=0$ and $b=L$, which is a sort of (complex) 'norm'. Note that, when applied to wavefunctions $\psi(x) \in \mathcal{L}^{2}$, it reminds the usual (real) norm in the Hilbert space albeit for replacing the squares by the square modulus and letting $a$ and $b$ recede respectively to $-\infty$ and to $+\infty$. For the resonant solutions, however, $N_{n}$ diverges in this limit.

\section{Inner products}

In this section, we shall calculate the usual inner products $\left\langle z_{n} \mid z_{m}\right\rangle$ of resonant states with themselves (7) and their mixed products $\left\langle z_{n} \mid E\right\rangle$ and $\left\langle\phi_{i} \mid z_{n}\right\rangle$ with the scattering and bound states respectively. The method also leads to the usual inner products $\left\langle\phi_{i} \mid \phi_{j}\right\rangle=\delta_{i j},\left\langle\phi_{i} \mid E\right\rangle=0$ and $\left\langle E \mid E^{\prime}\right\rangle=\delta\left(E-E^{\prime}\right)$.

\subsection{Resonances}

The space integral giving the inner product of two resonant solutions can be split in three sectors:

$$
\begin{aligned}
\left\langle z_{n} \mid z_{m}\right\rangle & \equiv \int_{-\infty}^{+\infty} \mathrm{d} x u_{n}^{*}(x) u_{m}(x) \\
& =R_{n}^{*} R_{m} \int_{-\infty}^{0} \mathrm{~d} x \mathrm{e}^{\mathrm{i}\left(p_{n}^{*}-p_{m}\right) x}+\int_{0}^{L} \mathrm{~d} x u_{n}^{*} u_{m}+T_{n}^{*} T_{m} \int_{L}^{\infty} \mathrm{d} x \mathrm{e}^{-\mathrm{i}\left(p_{n}^{*}-p_{m}\right) x} \\
& =\left(R_{n}^{*} R_{m}+T_{n}^{*} T_{m}\right) \int_{0}^{\infty} \mathrm{d} x \mathrm{e}^{-\mathrm{i}\left(p_{n}^{*}-p_{m}\right) x}+\int_{0}^{L} \mathrm{~d} x u_{n}^{*} u_{m}-T_{n}^{*} T_{m} \int_{0}^{L} \mathrm{~d} x \mathrm{e}^{-\mathrm{i}\left(p_{n}^{*}-p_{m}\right) x} .
\end{aligned}
$$




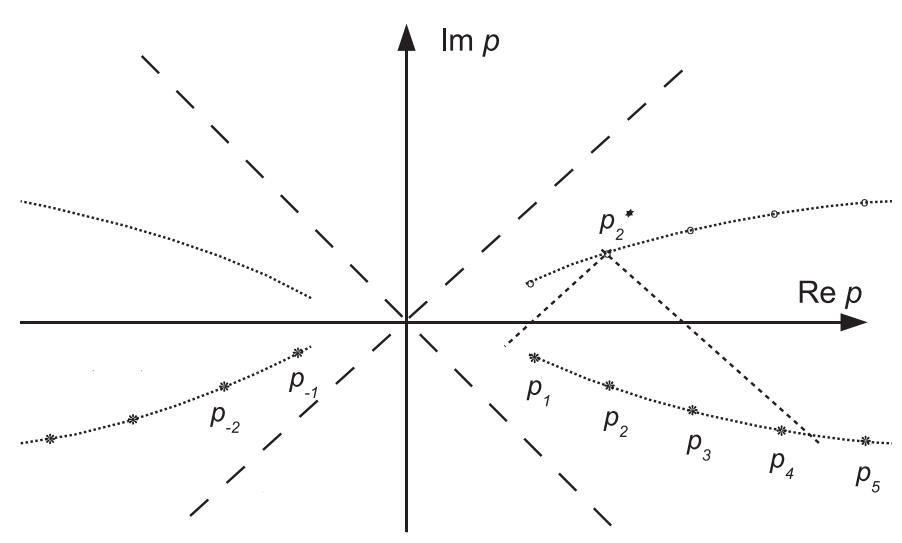

Figure 1. An example of orthogonality between resonant states: $\left\langle z_{2} \mid z_{n}\right\rangle=0$ for $n<0$ and $n>4$, while $\left\langle z_{2} \mid z_{m}\right\rangle=\infty$ for $0<m \leqslant 4$.

The ORBCs in 0 and $L$, together with their complex conjugates, allow us to express the finite integral $\int_{0}^{L} \mathrm{~d} x u_{n}^{*} u_{m}$ in terms of the amplitudes $R$ and $T$ outside the barrier, regardless of the explicit form of $u_{n}(x)$ inside the barrier and hence of the particular form of the potential. The procedure uses the Schrödinger operator $O \equiv-2 m H=\partial_{x}^{2}-2 m V$ and integration by parts to obtain

$$
\begin{aligned}
\left(p_{n}^{* 2}-p_{m}^{2}\right) \int_{0}^{L} \mathrm{~d} x u_{n}^{*} u_{m} & =2 m\left(z_{n}^{*}-z_{m}\right) \int_{0}^{L} \mathrm{~d} x u_{n}^{*} u_{m} \\
& =\int_{0}^{L} \mathrm{~d} x u_{n}^{*}(x)(\vec{O}-\overleftarrow{O}) u_{m}(x)=W\left[u_{n}^{*}, u_{m}\right]_{0}^{L}
\end{aligned}
$$

where $W[\phi, \psi] \equiv \phi \partial_{x} \psi-\psi \partial_{x} \phi$ is the Wronskian of the functions $\phi$ and $\psi$, so that

$$
\int_{0}^{L} \mathrm{~d} x u_{n}^{*} u_{m}=\frac{\mathrm{i}}{p_{n}^{*}-p_{m}}\left(T_{n}^{*} T_{m} \mathrm{e}^{-\mathrm{i}\left(p_{n}^{*}-p_{m}\right) L}+R_{n}^{*} R_{m}\right)
$$

Then cancellations occur such that

$$
\left\langle z_{n} \mid z_{m}\right\rangle=\left(R_{n}^{*} R_{m}+T_{n}^{*} T_{m}\right)\left(\int_{0}^{\infty} \mathrm{d} x \mathrm{e}^{-\mathrm{i}\left(p_{n}^{*}-p_{m}\right) x}+\frac{\mathrm{i}}{p_{n}^{*}-p_{m}}\right) .
$$

Now the point is the calculation of the integral in (13). We adopt the limit $\lambda \rightarrow 0$ in (29) as a prescription for the result of (13) and defer the discussion of our scheme to section 5 and to the appendix.

With this prescription, we finally obtain

$$
\left\langle z_{n} \mid z_{m}\right\rangle= \begin{cases}0, & -\frac{\pi}{4}<\arg \left(p_{m}-p_{n}^{*}\right)<5 \frac{\pi}{4} \\ \infty, & \text { otherwise. }\end{cases}
$$

In particular $\left\langle z_{n} \mid z_{n}\right\rangle=\infty$, as expected.

For each resonant state $\left|z_{n}\right\rangle$, the result (14) defines a 'neighbourhood of divergence' so that $\left|z_{n}\right\rangle$ is orthogonal to any other $\left|z_{m}\right\rangle$, the momentum $p_{m}$ of which lies outside a 'cone of divergence' with the apex in $p_{n}^{*}$, and gives a divergent inner product if $p_{m}$ lies inside this cone (figure 1). 


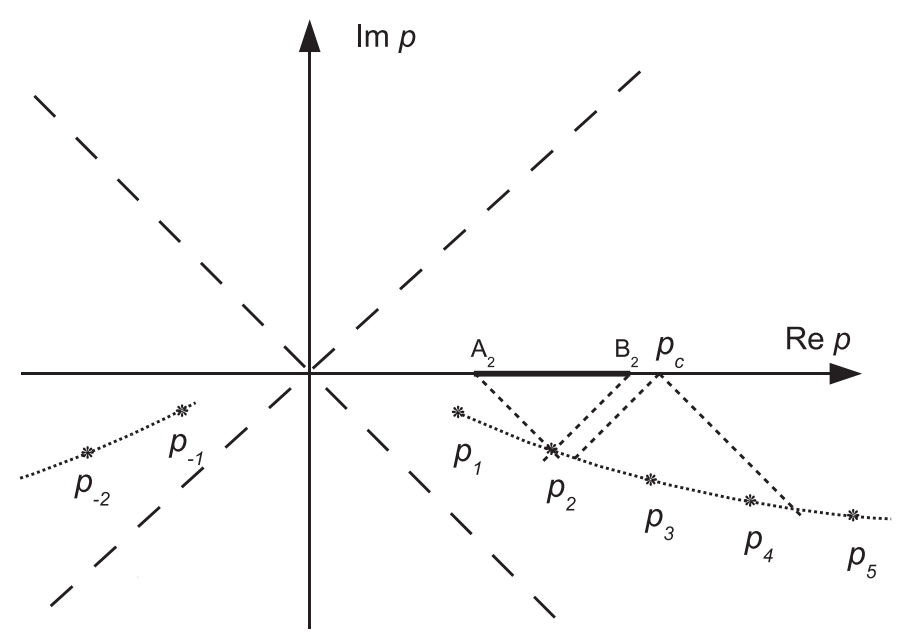

Figure 2. Two examples of orthogonality between resonant and scattering states: $\left\langle z_{2} \mid E\right\rangle=0(\infty)$ when $p \equiv \sqrt{2 m E} \subset \mathrm{R}_{+}$lies out of (inside) the interval $\left[A_{2}, B_{2}\right]$, and $\left\langle E_{c} \mid z_{n}\right\rangle=0(\infty)$ when $n \neq 3,4(n=3,4)$, respectively.

\subsection{Resonant and scattering states}

The in and out scattering solutions obey a single differential $\mathrm{BC}$ at one of the points $x=0$ or $x=L$. For instance, a right-moving in state obeys only the second BC in (4) with $p>0$. This is equivalent to imposing the asymptotic form

$$
\psi_{r}^{+}(x)= \begin{cases}\mathrm{e}^{\mathrm{i} p x}+R(p) \mathrm{e}^{-\mathrm{i} p x}, & x \leqslant 0 \\ T(p) \mathrm{e}^{\mathrm{i} p x}, & x \geqslant L .\end{cases}
$$

For instance, the combined BCs of the resonant and of the $(p>0$, in $)$ scattering solutions similarly lead to

$$
\begin{gathered}
\left\langle z_{n} \mid E\right\rangle=\int_{0}^{\infty} \mathrm{d} x\left(R_{n}^{*} \mathrm{e}^{-\mathrm{i}\left(p_{n}^{*}+p\right) x}+\left(R_{n}^{*} R+T_{n}^{*} T\right) \mathrm{e}^{-\mathrm{i}\left(p_{n}^{*}-p\right) x}\right) \\
+\frac{\mathrm{i}}{p_{n}^{*}-p}\left(R_{n}^{*} R+T_{n}^{*} T\right)+\frac{\mathrm{i}}{p_{n}^{*}+p} R_{n}^{*}
\end{gathered}
$$

With $p>0$, for $n>0$ we always have $-\frac{\pi}{4}<\arg \left(p_{n}^{*}+p\right)<5 \frac{\pi}{4}$, so that

$$
\left\langle z_{n} \mid E\right\rangle=\left(R_{n}^{*} R+T_{n}^{*} T\right)\left(\int_{0}^{\infty} \mathrm{d} x \mathrm{e}^{-\mathrm{i}\left(p_{n}^{*}-p\right) x}+\frac{\mathrm{i}}{p_{n}^{*}-p}\right)
$$

and therefore, with the prescription adopted,

$$
\left\langle z_{n} \mid E\right\rangle= \begin{cases}0, & -\frac{\pi}{4}<\arg \left(p-p_{n}^{*}\right)<5 \frac{\pi}{4} \\ \infty, & \text { otherwise. }\end{cases}
$$

This means that a given scattering in state $|E\rangle$ (with momentum $p>0$ on the real axis) is orthogonal to any $\left|z_{n}\right\rangle$ (with $n>0$ ) if the momentum $p_{n}$ lies outside the cone with the apex in $p$, the inner product being divergent otherwise. Vice versa, given $p_{n}$, the momenta $p$ of the orthogonal scattering states lie outside the cone with the apex in $p_{n}$ (figure 2).

The scattering states always have a reflected wave with a momentum of opposite sign to the incident one, so the situation is trickier for $n<0$. Given $p_{-|n|}$, the cone to be considered is again the one with apex in the mirror momentum $p_{|n|}$. 


\subsection{Resonant and bound states}

Let us suppose that, besides the barrier, there is some potential well within the region $[0, L]$ sustaining the bound states $\left|\phi_{i}\right\rangle$ with purely imaginary momenta $p_{i}=\mathrm{i} q_{i}\left(q_{i}>0\right)$. The exponential decrease of the amplitude outside the well, corresponding to resonant-like BCs

$$
\left.\partial_{x} \phi\right|_{x=0}=q \phi(0),\left.\quad \partial_{x} \phi\right|_{x=L}=-q \phi(L) \quad(q>0),
$$

which imply the more popular (and weaker) $\phi_{i}( \pm \infty)=0$, may be translated into the assumption of an asymptotic form similar to (5), namely

$$
\phi_{i}(x)= \begin{cases}R_{i} \mathrm{e}^{q_{i} x}, & x \leqslant 0 \\ T_{i} \mathrm{e}^{-q_{i} x}, & x \geqslant L\end{cases}
$$

which is manifestly square integrable.

Following the same steps leading to (13), we now have

$$
\left\langle\phi_{i} \mid z_{n}\right\rangle=\left(R_{i}^{*} R_{n}+T_{i}^{*} T_{n}\right)\left(\int_{0}^{\infty} \mathrm{d} x \mathrm{e}^{\mathrm{i}\left(\mathrm{i} q_{i}+p_{n}\right) x}-\frac{\mathrm{i}}{\mathrm{i} q_{i}+p_{n}}\right) .
$$

The integral is convergent, yielding $\mathrm{i}\left(\mathrm{i} q_{i}+p_{n}\right)^{-1}$ and hence $\left\langle\phi_{i} \mid z_{n}\right\rangle=0$, provided that $q_{i}>\left|\operatorname{Im} p_{n}\right|$. Thus, we have a situation similar to that occurring between the resonances, namely that the states are orthogonal if the bound state momentum, lying in the positive imaginary axis, and the resonant one, lie outside the respective divergence cones, the inner product being infinite otherwise. It might happen that, for general analyticity reasons for any general potential $V(x)$ with barriers and wells within $[0, L]$, the cones of the allowed bound state momenta do never include the resonant momenta, but this point would require separate investigation.

\section{Symmetrical inner products}

Together with the standard definition (7), which yields (finite or infinite) real norms, we shall also consider the alternative symmetrical definition

$$
\{\Phi \mid \Psi\} \equiv \int_{-\infty}^{+\infty} \mathrm{d} x \phi(x) \psi(x),
$$

which arises in the convolution of kernel (8) with the initial state, and yields complex 'norms'.

The calculation follows the same lines as above relying on the boundary conditions:

$$
\left\{z_{n} \mid z_{m}\right\}=\left(R_{n} R_{m}+T_{n} T_{m}\right)\left(\int_{0}^{\infty} \mathrm{d} x \mathrm{e}^{\mathrm{i}\left(p_{n}+p_{m}\right) x}-\frac{\mathrm{i}}{p_{n}+p_{m}}\right) .
$$

The result is

$$
\left\{z_{n} \mid z_{m}\right\}= \begin{cases}0, & -\frac{\pi}{4}<\arg \left(p_{m}+p_{n}\right)<5 \frac{\pi}{4} \\ \infty, & \text { otherwise. }\end{cases}
$$

For a given $\left|z_{n}\right\rangle$, the apex of the divergence cone is at $-p_{n}$ so that, in particular, $\left\{z_{n} \mid z_{m}\right\}=0$ if both $m, n>0$, also if both $m, n<0$, and, more noteworthy, $\left\{z_{n} \mid z_{n}\right\}=0$ for any $n$. Only some of the products are divergent when $n$ and $m$ have opposite sign.

For the crossed products of $\left|z_{n}\right\rangle(n>0)$ with the scattering $p>0$ in state, one has

$$
\left\{z_{n} \mid E\right\}=\int_{0}^{\infty} \mathrm{d} x\left(R_{n} \mathrm{e}^{\mathrm{i}\left(p_{n}-p\right) x}+\left(R_{n} R+T_{n} T\right) \mathrm{e}^{\mathrm{i}\left(p_{n}+p\right) x}\right)-\frac{\mathrm{i}}{p_{n}+p}\left(R_{n} R+T_{n} T\right)-\frac{\mathrm{i}}{p_{n}-p} R_{n} .
$$


One always has $-\frac{\pi}{4}<\arg \left(p_{n}+p\right)<5 \frac{\pi}{4}$, so that

$$
\left\{z_{n} \mid E\right\}= \begin{cases}0, & -\frac{\pi}{4}<\arg \left(p_{n}-p\right)<5 \frac{\pi}{4} \\ \infty, & \text { otherwise. }\end{cases}
$$

Therefore, the divergence cone has the apex in $p_{n}$, as in the case of the standard inner product $\left\langle z_{n} \mid E\right\rangle$. For $n<0$, only $p+p_{-|n|}$ may lie in the sector $5 \frac{\pi}{4}<\arg \left(p_{n}+p\right)<7 \frac{\pi}{4}$ leading to a divergent product, so that, for a given $p_{-|n|}$ the $p$-states are orthogonal if $p$ lies inside the cone with apex in the mirror momentum $p_{|n|}$, reproducing the same situation encountered for the standard product.

Because of the relationship $u_{-n}(x)=u_{n}^{*}(x)$ between the outgoing solutions, we have $\left\langle z_{n} \mid z_{m}\right\rangle=\left\{z_{-n} \mid z_{m}\right\}$ and $\left\{z_{n} \mid z_{m}\right\}=\left\langle z_{-n} \mid z_{m}\right\rangle$. Then the result $\left\{z_{n} \mid z_{n}\right\}=0$ is less surprising if rewrite it as $\left\langle z_{-n} \mid z_{n}\right\rangle=0$, just a particular case of the conventional orthogonality between states with momenta in opposite quadrants of the lower half complex plane.

\section{Regularization of the divergent products}

Several proposals have been worked out in the literature to deal with the divergent inner products. We quote here the one by Romo [11] of analytically continuing the momentum dependence of the solutions $u(x)$ inside the products from the upper half complex plane, where the (outgoing) function would be square-integrable, to the lower half-plane where the resonant momenta lie. This is equivalent to prescribe the finite value $\mathrm{i} k^{-1}$ for the result of the integral $\int_{0}^{\infty} \mathrm{d} x \mathrm{e}^{\mathrm{i} k x}$ also in the whole lower half-plane $k$, where it would actually be divergent (see the discussion in the appendix). This approach, combined with advantages of working in momentum representation, has been followed in later works [18].

Other proposals rely on the introduction of convergence factors in the integral which are able to cope with the asymptotic exponential growth of the resonant solutions. The simple factor $\mathrm{e}^{-\lambda x},(\lambda$ real $>0)$ works only if $\lambda$ is greater than the absolute values of the imaginary parts of given $p_{n}$ and $p_{m}$ (which grow with increasing $n, m$ ), so that $\lambda$ cannot be brought to zero without rendering divergent the integral. However, it lets performing an analytic continuation in $\lambda$ to its 'forbidden' values which may be adopted as a prescription.

Following Zel'dovich [13], we adopt the more powerful Gaussian factor $\mathrm{e}^{-\lambda x^{2}}$, which is able to overcome the growth of all the resonant solutions even for $\lambda \rightarrow 0_{+}$, and is computationally tractable $[5,13]$ :

$$
\left\langle\left\langle z_{n} \mid z_{m}\right\rangle\right\rangle_{\lambda} \equiv \int_{-\infty}^{+\infty} \mathrm{d} x \mathrm{e}^{-\lambda x^{2}} u_{n}^{*}(x) u_{m}(x) \quad(\lambda \text { real }>0) .
$$

The functions $\mathrm{e}^{-\frac{1}{2} \lambda x^{2}} u_{n}(x)$ are not the eigenfunctions of the Hamiltonian, so the exact calculation of (27) requires the knowledge of the explicit form of the solutions inside the barrier.

Equation (10) now reads

$$
\begin{aligned}
\left\langle\left\langle z_{n} \mid z_{m}\right\rangle\right\rangle_{\lambda}=( & \left.R_{n}^{*} R_{m}+T_{n}^{*} T_{m}\right) \int_{0}^{\infty} \mathrm{d} x \mathrm{e}^{-\lambda x^{2}} \mathrm{e}^{-\mathrm{i}\left(p_{n}^{*}-p_{m}\right) x} \\
& +\int_{0}^{L} \mathrm{~d} x \mathrm{e}^{-\lambda x^{2}} u_{n}^{*} u_{m}-T_{n}^{*} T_{m} \int_{0}^{L} \mathrm{~d} x \mathrm{e}^{-\lambda x^{2}} \mathrm{e}^{-\mathrm{i}\left(p_{n}^{*}-p_{m}\right) x} .
\end{aligned}
$$


According to (A.1), this regularized expression is finite for $\lambda>0$ :

$$
\begin{aligned}
\left\langle\left\langle z_{n} \mid z_{m}\right\rangle\right\rangle_{\lambda}=( & \left.R_{n}^{*} R_{m}+T_{n}^{*} T_{m}\right) \frac{-\mathrm{i}}{p_{n}^{*}-p_{m}} \sqrt{\pi} z \mathrm{e}^{z^{2}} \operatorname{erfc}(z) \\
& +\int_{0}^{L} \mathrm{~d} x u_{n}^{*} u_{m}-T_{n}^{*} T_{m} \int_{0}^{L} \mathrm{~d} x \mathrm{e}^{-\mathrm{i}\left(p_{n}^{*}-p_{m}\right) x}+O(\lambda)
\end{aligned}
$$

where $z=\mathrm{i}\left(p_{n}^{*}-p_{m}\right) /(2 \sqrt{\lambda})$

In the limit $\lambda \rightarrow 0_{+}$, the terms $O(\lambda)$ vanish, and for $\sqrt{\pi} z \mathrm{e}^{z^{2}} \operatorname{erfc}(z)$, we have the result (A.5). Thus, we obtain (14) in this limit, which must be intended as a prescription for the calculation of (13). We discuss this issue in more detail in the appendix.

Note that for finite $\lambda>0$, the exact integrals over the finite interval $[0, L]$ get rather involved and the one over the resonant functions inside the barrier depends on the explicit form of the functions other than on the position of the resonant momenta.

The regularization works in a similar fashion for the crossed inner products $\left\langle z_{n} \mid E\right\rangle$ and $\left\langle z_{n} \mid \phi_{i}\right\rangle$, as well as for the symmetrical definition.

\section{Conclusions}

We have calculated some relevant inner products involving the resonant eigenstates in the example of a one-dimensional quantum potential barrier with compact support. This old problem is non-trivial since the modulus of the resonant solutions grows exponentially at the spatial infinity, giving rise to infinite norms and seemingly infinite inner products.

Among the variety of historical proposals to circumvent these difficulties, we have adopted a Gaussian convergence factor, first introduced by Zel'dovich, and carried out the limit of the integrals where the factor fades off to unity. This prescription yields inner products such that most of these states are orthogonal to each other, except when they lie in a neighbourhood defined by a 'divergence cone', in which case the product is infinite. Similarly, scattering states (with real momentum $p>0$ ) and bound states (pure imaginary momenta $\mathrm{i} q_{i}$, with $q_{i}>0$ ) are orthogonal to a given resonant state $\left|z_{n}\right\rangle$ with the momentum $p_{n}$, except when $p$ or i $q_{i}$ lie in a neighbourhood of $p_{n}$. Thus, the resonant states share the properties of the continuum and discrete real spectra, namely (partial) orthogonality and the infinite norm characteristic to the Dirac states.

This result is different from the full bi-orthogonality obtained by the prescription of analytically continuing the finite integrals from the upper complex momentum plane to the whole lower half-plane, where the resonant momenta lie but where the integrals are formally divergent. Our limiting procedure instead extends the finite result to the more modest $\pi / 4$ angular sectors of the lower half-plane close to the real axis. We differ also from earlier attempts [5] using the Gaussian convergence factor, actually limited to the products $\left\langle z_{-n} \mid z_{m}\right\rangle$ (where $\left|z_{-n}\right\rangle$ was mistaken for the incoming state $\left|\tilde{z}_{n}\right\rangle$ ), in that our exact calculation also yields $\left\langle z_{-n} \mid z_{n}\right\rangle=0$ instead of a finite quantity normalizable to 1 . The comparison with the results obtained in the Friedrichs model [19, 20] is also interesting.

Therefore, the orthogonality and normalization properties obtained depend on the prescription adopted, although one should expect, as a signature of their consistency, that all of them lead to the same unique result. For instance, the inner self-product $\left\langle z_{n} \mid z_{n}\right\rangle$ yielding the square of the conventional norm $\left\|z_{n}\right\|$, which is manifestly divergent on the same footing of the scattering states, is finite for some prescriptions, whereas ours recovers the infinite result.

We do not investigate here the question if a regularization by different convergence factors (using for instance $\mathrm{e}^{-\lambda|x|^{v}}$ for some real value $1<v<2$ ) would yield narrower divergence cones, thus approaching the result $\left\langle z_{n} \mid z_{m}\right\rangle=0(n \neq m)$ which would seem more natural 
and closer to the result of the analytical continuation prescription. On the other hand, such a continuation in the momentum of the solutions is a non-trivial matter [21] related to the two-sheet structure of the complex energy plane.

The completeness of the scattering (plus bound, if any) solutions in the Hilbert space is not inherited by the sole resonant (plus bound) states in resonance expansions of the unity, and a continuum of complex energy 'background' states must be included [5, 22,23]. Any consistent unambiguous prescription for the calculation of the inner products involving resonances should be tested against the requirement of idempotence of the unity, as discussed in the Introduction. It is not clear how could it happen in the analytical continuation prescriptions, where the claimed orthogonality between resonances (or between resonant and anti-resonant states) is of the Kronecker- $\delta$ type, and the cross inner products between bound, resonant and background states have not been worked out.

We instead have orthogonality between most of the states and divergent inner products between 'neighbour' resonant (and continuum and bound) states, which then have also infinite self-products consistently with the well-known infinite norm of the resonances. In a general expansion involving bound, resonant (in variable number, according to the poles encircled by the deformed integration path), scattering and continuum background states, showing directly the idempotence of the unity requires tackling the products involving all of these families of states. In this paper, we have done part of this program and developed some regularization and calculational techniques. Work is in progress on the inner products involving the resonant and the background states, aiming to the eventual cancellation of the divergences encountered.

\section{Acknowledgments}

Work supported by MEC project FIS2008-05705. The authors are indebted to J León for suggestions and helpful discussions. J Julve acknowledges the hospitality of the Dipartimento di Fisica dell'Università di Bologna, Italy, where part of this work was done.

\section{Appendix. Limits of $\operatorname{erfc}(z)$-related integrals}

We rely on the basic integral

$$
J(k, \lambda) \equiv \int_{0}^{+\infty} \mathrm{d} x \mathrm{e}^{-\lambda x^{2}} \mathrm{e}^{\mathrm{i} k x}=\frac{\mathrm{i}}{k} \sqrt{\pi} z \mathrm{e}^{z^{2}} \operatorname{erfc}(z) \quad(\lambda \text { real }>0)
$$

which is directly related to (7.1.2) in [24], where $z=-\mathrm{i} k /(2 \sqrt{\lambda})$, and hence $k$ can take any complex value. Note that on completing a square in the exponent and changing the integration variable to $t=\sqrt{\lambda} x+z$, one obtains the intermediate expression

$$
J(k, \lambda)=\frac{1}{\sqrt{\lambda}} \mathrm{e}^{z^{2}} \int_{z}^{\infty+z} \mathrm{~d} t \mathrm{e}^{-t^{2}}
$$

The integral representation (7.1.2) is convergent when the path of the complex integration variable $t$ approaches $\infty$ along a direction $-\frac{\pi}{4}<\arg (t)<\frac{\pi}{4}$. This condition is fulfilled by (A.2) as long as $z$ remains finite. However, in (A.1) the limit $\lambda \rightarrow 0$ (and hence $z \rightarrow \infty$ ) can not always be taken before the integration is carried out because the function $f_{\lambda}(x) \equiv \mathrm{e}^{-\lambda x^{2}}$ does not converge uniformly to the function $f_{0}(x)=1$ when $\lambda \rightarrow 0$.

For Im $k>0$, the limit can be taken in the integrand in (A.1) because the finiteness of the integral is always assured by the convergence factor $\mathrm{e}^{-(\operatorname{Im} k) x}$, trivially yielding $\mathrm{i} k^{-1}$. In this case, $J(k, \lambda)$ converges to $J(k, 0)=\mathrm{i} k^{-1}$ when $\lambda \rightarrow 0$. 
For real $k$, the result

$$
\int_{0}^{\infty} \mathrm{d} x \mathrm{e}^{\mathrm{i} k x}=\mathrm{i} P V \frac{1}{k}+\pi \delta(k)
$$

is also well known. Note that it relies on adding to $k$ a small imaginary part $\mathrm{i} \epsilon$, which still guarantees the convergence when $\lambda \rightarrow 0$, but later in the limit $\epsilon \rightarrow 0_{+}$the result must be interpreted as a distribution.

For $\operatorname{Im} k<0$, the integration and the limit $\lambda \rightarrow 0$ do not commute. In that case, we adopt the limit of the integral as a prescription. On the right-hand side of (A.1), the limit $\lambda \rightarrow 0$ can be directly inferred from the asymptotic expansion

$$
\sqrt{\pi} z \mathrm{e}^{z^{2}} \operatorname{erfc}(z) \sim 1+\sum_{m=1}^{\infty}(-1)^{m} \frac{1 \cdot 3 \ldots(2 m-1)}{\left(2 z^{2}\right)^{m}} \quad\left(z \rightarrow \infty,|\arg z|<\frac{3 \pi}{4}\right)
$$

(see (7.1.23) in [24]), namely

$$
\lim _{z \rightarrow \infty} \sqrt{\pi} z \mathrm{e}^{z^{2}} \operatorname{erfc}(z)= \begin{cases}1, & -3 \frac{\pi}{4}<\arg (z)<3 \frac{\pi}{4} \\ \infty, & \text { otherwise }\end{cases}
$$

which can also be numerically checked. In this way, (A.5) yields an extension of $J(k, 0)$ to a new region of the lower half complex plane:

$$
J(k, 0)= \begin{cases}\frac{\mathrm{i}}{k}, & -\frac{\pi}{4}<\arg (k)<5 \frac{\pi}{4}, \quad k \neq 0 \\ \infty, & \text { otherwise. }\end{cases}
$$

\section{References}

[1] Nussenzveig H M 1972 Causality and Dispersion Relations (New York and London: Academic)

[2] Hatano N, Kawamoto T and Feinberg J 2009 Probabilistic interpretation of resonant states arXiv:0904.1044

[3] Gamow G 1928 Z. Phys. 51271

[4] Siegert G 1939 Phys. Rev. A 56750

[5] Berggren T 1968 Nucl. Phys. A 109265

[6] Newton R G 1960 J. Math. Phys. 1319

[7] García-Calderón 1976 Nucl. Phys. A 261130

[8] García-Calderón 1982 Lett. Nuovo Cimento 33253

[9] Julve J and Urríes F J 2008 J. Phys. A: Math. Theor. 41304010

[10] García-Calderón G, Romo R and Villavicencio J 2009 Phys. Rev. A 79052121

[11] Romo W J 1968 Nucl. Phys. A 116618

[12] Zavin R and Moiseyev N 2004 J. Phys. A: Math. Gen. 374619

[13] Zel'dovich Ya B 1961 Sov. Phys._JETP 12542

[14] García-Calderón G and Peierls R 1976 Nucl. Phys. A 265443

[15] Bohm A and Gadella M 1989 Lecture Notes in Physics vol 348 (Berlin: Springer)

[16] de la Madrid R and Gadella M 2002 Am. J. Phys. 70626

[17] García-Calderón G and Rubio A 1997 Phys. Rev. A 553361

[18] Hernández E and Mondragón A 1984 Phys. Rev. C 29722

[19] Antoniou I E and Prigogine I 1993 Physica A 192443

[20] Civitarese O and Gadella M 2004 Phys. Rep. 39641

[21] de la Madrid R 2009 SIGMA 70626

[22] Berggren T 1982 Nucl. Phys. A 389261

[23] de la Madrid R, García-Calderón G and Muga G 2005 Czech. J. Phys. 551141

[24] Abramowitz M and Stegun I A 1965 Handbook of Mathematical Functions (New York: Dover) p 297 